\title{
Phospholipid Metabolism
}

National Cancer Institute

\section{Source}

National Cancer Institute. Phospholipid Metabolism. NCI Thesaurus. Code C40656.

Phospholipid Metabolism involves anabolic and catabolic biochemical changes made to phospholipids. 